\title{
Heuristics and representational change in two-move matchstick arithmetic tasks
}

\author{
Michael Öllinger ${ }^{1}$, Gary Jones ${ }^{2}$, and Günther Knoblich ${ }^{3}$
}

\author{
${ }_{1}^{1}$ Parmenides Center for the Study of Thinking, Munich, Germany \\ 2 Psychology Department, Nottingham Trent University, Burton Street, Nottingham, NG1 4BU, UK \\ 3 Psychology Department, Rutgers University, 101 Warren Street, Newark, NJ 07102
}

Received 30.03.2006

Accepted 16.08.2006

\section{Keywords}

insight, heuristics, representational change

\section{ABSTRACT}

Insight problems are problems where the problem solver struggles to find a solution until * aha! * the solution suddenly appears. Two contemporary theories suggest that insight problems are difficult either because problem solvers begin with an incorrect representation of the problem, or that problem solvers apply inappropriate heuristics to the problem. The relative contributions of representational change and inappropriate heuristics on the process of insight problem solving was studied with a task that required the problem solver to move two matchsticks in order to transform an incorrect arithmetic statement into a correct one. Prob- lem solvers ( $N=120)$ worked on two different types of two-move matchstick arithmetic problems that both varied with respect to the effectiveness of heuristics and to the degree of a necessary representational change of the problem representation. A strong influence of representational change on solution rates was found whereas the influence of heuristics had minimal effects on solution rates. That is, the difficulty of insight problems within the twomove matchstick arithmetic domain is governed by the degree of representational change required. A model is presented that details representational change as the necessary condition for ensuring that appropriate heuristics can be applied on the proper problem representation.

\section{INTRODUCTION}

Consider the anecdote of King Hiero asking Archimedes to prove that the amount of gold in his newly made crown equals the amount of gold given to the goldsmiths. Archimedes considers the problem for some time, and becomes stuck in an impasse - he simply cannot see a solution. Some days later when taking a bath, he notices that his body displaces the water in the bath tub. Immediately, he has his flash of insight and runs naked through the streets, crying out "Eureka! - I have found it" (Gruber, 1995). Archimedes clearly had the necessary knowledge to solve the problem, so why was it so hard for him to gain his insight? How was he able to overcome the impasse in which he was stuck?
Insight is still a "mysterious" phenomenon within problem solving literature. Currently, there are at least two theoretical accounts that try to explain the processes involved in insight problem solving in order to "demystify insight" (Bowden et al. 2005): The first stresses the importance of heuristics (MacGregor, Ormerod, \& Chronicle, 2001); the second stresses the necessity of a representational change (Ohlsson, 1992). In this paper we make a further attempt to systematically disentangle the influence of heuristics

Correspondence concerning this article should be addressed to Parmenides Center for the Study of Thinking Kardinal Faulhaberstr. 14 a, 80333 Munich, Germany Phone: ++49 (89) 21022914 Fax: ++49 (89) 21022929 Email: michael.oellinger@parmenides-foundation.org 
and representational change by assessing whether the difficulty in solving insight problems is due to the search for proper heuristics that can be applied or whether it is due to requiring a change of the initial problem representation into a problem representation that includes the solution (cf. Jones, 2003). The role of heuristics in problem solving will now be examined, before covering the insight literature in relation to heuristics and to representational change. The goals of the current paper will then be outlined.

\section{Heuristics in problem solving}

No one would seriously entertain doubts about the importance of heuristics for problem solving (Gigerenzer \& Todd, 1999). Most current theorists consider heuristics, or rules of thumb, to be the critical operators for the solution of problems (Kaplan \& Simon, 1990; Mayer, 1992; Lovett \& Anderson, 1996; Anderson \& Lebiere, 1998). In particular, the information processing account has strongly emphasized and focused on the role of heuristics (e.g., hill climbing, or means-end heuristics) as the key for understanding the human problem solving process - and this has been demonstrated successfully for a variety of problems (e.g., Newell \& Simon, 1972).

Newell and Simon's (1972) information processing account of problem solving suggests that problem solvers initially generate an internal representation, the problem representation, of the given problem, or task - the initial state. Problem solving involves applying operators to transform the initial state into the desired goal state, with a multitude of possible intermediary states being created along the solution path. The closed set of all possible states of a problem is termed the problem space. Within the problem space there are more or less direct paths from the initial state through to the goal state. Newell and Simon suggest that heuristics are used to help the problem solver navigate the problem space by the selection of effective operators that reduce the distance to the goal much more effectively than blind trial-and-error. As a general rule, Problem Space Theory suggests that the larger the problem space, the more difficult the problem.

The most important heuristics are hill-climbing and means-ends analysis (Newell \& Simon, 1972; Greeno, 1974; Thomas, 1974; Anderson \& Lebiere, 1998; Chronicle, MacGregor \& Ormerod, 2004). In hill-climbing, the problem solver selects operators that make the current state of the problem as similar as possible to the required goal state. As a result, there is a tendency to select moves that reduce the distance between the current and goal states as much as possible. This pattern was consistently found in several empirical studies (Greeno, 1974; Atwood \& Polson, 1976; Chronicle et al., 2004). In means-ends analysis, the problem solver reduces the problem into sub-goals and continues to create sub-goals until an available operator (means) can be applied. Sub-goals are worked off in a stepwise manner until the desired goal is attained.

Although many problems can be described, analyzed and solved based on the assumptions of Problem Space Theory (Newell and Simon used cryptarithmetic problems and the Tower of Hanoi problem), these tend to be problems that are well-defined (i.e., where it is clear what the initial state, goal state, and possible moves can be). In general, the more ill-defined a problem is, the more difficult it is to explain in Problem Space Theory, because it becomes difficult to derive the problem space and hence apply heuristics to reduce the problem space. This is often the case for insight problems, which are often regarded as ill-defined. However, some insight problems are difficult, even though they are well-defined with clear initial and goal states, and with all of the available operators to change states in the problem also being clear. Furthermore, insight problems frequently have a small problem space. Problem Space Theory alone cannot account for insight problem solving phenomena, because its principles do not explain why people reach an impasse on clearly defined problems with a small problem space, and why they require an insight in order to solve them. Hitherto only a few attempts have been made to provide extensions for the Problem Space Theory in terms of insight problem solving.

One possible route of explanation involves the use of heuristics, suggesting that a potential failure to gain insight is due to the inappropriate application of heuristics (MacGregor, Ormerod, \& Chronicle, 2001). Alternatively, another route of explanation is that the problem solver fails to generate an adequate problem representation, and thus representational change is required in order to see the solution (Ohlsson, 1992). We will now review the current thinking with respect to whether insight arises from people applying inappropriate heuristics or whether arises from people beginning problems with an incorrect problem representation.

\section{Insight: Inappropriate representation or inappropriate heuristics?}

In terms of solving insight problems, solutions are: (i) accompanied by an "Aha!" experience 
(Bühler, 1907); (ii) sudden in appearance (Novick \& Sherman, 2003; Jung-Beeman et al., 2004, Bowden et al. 2005); (iii) unintended (Wegner, 2002); (iv) not stepwise (Metcalfe, 1986a, 1986b; Metcalfe \& Wiebe, 1987).

Aside from this phenomenological description of insight, there are a number of researchers who propose that insight problems are special because they require a representational change (Wertheimer, 1959; Kaplan \& Simon, 1990; Ohlsson, 1992; Sternberg \& Davidson, 1995; Knoblich et al., 1999). According to this view, accomplishing a change in one's representation is the main source of difficulty in achieving insight. Representational change is an extension of the Gestalt term "Restructuring" (see Ohlsson, 1984a, 1984b), which states that either a change of the given constituents of the problem representation ("seeing" the problem in another way) or a change of the goal representation is necessary to achieve insight.

For example, an inappropriate problem representation that over-constrains the search space for a possible solution could be to search for a solution within a $2 \mathrm{D}$ space when a $3 \mathrm{D}$ representation is required (e.g., the six-matchstick-problem introduced by Katona, 1940). That is, the problem solver's goal representation only enables the selection of operators that move matchsticks in the 2D plain (Isaak \& Just, 1995).

According to Ohlsson's Representational Change Theory, representational change is not the result of a deliberate search, but the result of unconscious processes that occur when problem solvers get stuck in an impasse. An impasse is defined as a mental state where problem solving behavior ceases and the problem solver does not know what to do next. To overcome an impasse a representational change is necessary. The trigger for a representational change is repeated failure due to the constraints of the initial problem representation.

One unconscious process that relaxes self-imposed constraints induced by problem solvers' prior knowledge is termed constraint relaxation. Constraint relaxation extends the goal representation of the problem solver - e.g., relaxing the 2D constraint in the Katona problem and searching in 3D space for a solution. That is, the problem solvers' problem space extends and as a result new solution options are available. Empirical evidence has shown that constraint relaxation is important in the solving of insight problems across a variety of domains, such as matchstick arithmetic (Knoblich et al., 1999; Knoblich, Ohlsson \& Raney, 2001; Öllinger \&
Knoblich, 2003), the car-park-game (Jones, 2003), and the tumor-problem (Grant \& Spivey, 2003). Moreover, the principle can be applied to explain the difficulty of many classical insight problems (Ohlsson, 1992; Isaak \& Just, 1995).

An alternative position specifies that the problem difficulty of insight problems depends on the inappropriate application of heuristics (MacGregor, Ormerod \& Chronicle, 2001; Ormerod, MacGregor \& Chronicle, 2002; Chronicle et al., 2004). The Criterion for Satisfactory Progress Theory (MacGregor et al., 2001) focuses on the role heuristics play within the problem solving process using a means-ends analysis framework. For the Criterion for Satisfactory Progress Theory, the difficulty of insight problems is not because of an inappropriate problem representation, but due to the application of inappropriate heuristics, a view that holds close to the assumptions of the original Problem Space Theory. The Criterion for Satisfactory Progress Theory suggests that problem solving follows two basic principles: (a) problem solvers seek to maximize each move such that the move results in a state which is as close as possible to the desired goal (hill-climbing); (b) problem solvers monitor their progress and only select moves that meet the criterion of progress - when a selected move fails to meet the criterion, there is an impulse to seek alternative solutions (c.f. Ormerod et al., 2002, p. 792).

Maximization and progress monitoring are thus necessary in order for insightful moves to be sought, and these processes trigger the discovery and retention of so-called promising states that meet the progress monitoring criterion and as a consequence attenuate the problem space. Furthermore, promising states lead to the realization that novel moves are of potential value and as a consequence a re-conceptualisation of the problem space is possible.

The probability of meeting an impasse also varies based on a person's lookahead value. Lookahead is determined by the capacity of potential moves a person can "look ahead" and hold in mind, which varies across individuals. Insight will be sought more quickly for people with a high lookahead capacity because they will realize more quickly that the problem at hand cannot be solved by the initially applied heuristics. MacGregor and colleagues have successfully applied the assumptions of maximization, progress monitoring and lookahead on the nine-dot problem and the eightcoin problem (MacGregor et al., 2001; Ormerod et al., 
2002), and recently on a set of other coin problems (Chronicle et al., 2004).

In summary, the Criterion for Satisfactory Progress Theory assumes that insight problems are difficult because the problem solver applies the wrong heuristics on the given problem representation, whereas the Representational Change Theory claims that the problem solver manipulates an inadequate problem representation.

\section{The present study}

Both theories (Representational Change Theory and Criterion for Satisfactory Progress Theory) follow the tradition of the Problem Space Theory and both try to extend this account to explain insight problem solving. However, the Representational Change Theory assumes that a representational change is responsible for overcoming an impasse, whereas the Criterion for Satisfactory Progress Theory postulates that the realisation of applying an inappropriate heuristic is the trigger for overcoming an impasse. As Jones (2003) demonstrated for the car-park game and Kershaw and Ohlsson (2004) showed for the nine-dot problem, it may not be helpful to polarize these theoretical approaches, since both of them are incapable of explaining the entire problem solving process for insight problems. Therefore, it seems more fruitful to identify what aspects of insight problems contribute to the overall problem difficulty, and investigate which parts of each theory best explain these contributions.

The goal of this study is to see whether the main source of difficulty in insight problems arises from the need to change the problem representation or from the use of inappropriate heuristics. In order to examine this, a paradigm was developed in which the degree of representational change and the effectiveness of heuristics can be systematically varied at the same time.

\section{THE TASK}

The task is an adaptation of the matchstick arithmetic problems used by Knoblich and colleagues (Knoblich et al., 1999; Knoblich et al., 2001; Öllinger \& Knoblich, 2003). The assumptions of the Representational Change Theory have been empirically validated by this task. Within the matchstick arithmetic task there are well defined insight problems where it is possible to determine the problem space (initial state, intermediate states, goal state). The great advantage of the matchstick arithmetic task is that problems can be constructed that have a similar outline, but that require differing degrees of representational change.

The basic matchstick arithmetic domain presents the problem solver with a roman numeral equation such as IV - III = III with the task of moving one matchstick to make the equation valid. According to the Representational Change Theory such a problem activates, according to prior knowledge, a goal representation that represents the values of the equation as variable, whereas the operators $(+,-$, and $=$ ) are represented as constants (e.g.: Var $_{1}$ const $_{1}$ Var $_{2}$ const $_{2}$ $\mathrm{Var}_{3}$, the prior knowledge hypothesis). This view has been supported empirically, with people's initial eye movements being focused on the values in the equations rather than the operators (Knoblich et al., 2001). As a consequence, problem solvers will try to manipulate the values to attain the goal (in the example, the I of the IV is moved after the V to make VI - III = III), but they do not try to manipulate the operators of the equation.

The same goal representation is established for the equation VI = III - III. Again, problem solvers will manipulate the values, but they will fail with this strategy. This problem requires a relaxation of the over-constrained goal representation which has to be changed to $\operatorname{Var}_{1} \mathrm{OP}_{1} \operatorname{Var}_{2} \mathrm{OP}_{2} \operatorname{Var}_{3}$. That is, the operators are also considered to be variable and therefore the possible search space for a solution becomes larger (in the example, one stick of the equal sign is moved into the minus sign VI - III = III). The extension of the problem solver's initial problem representation marks the moment of insight. Actually, Knoblich and colleagues (1999, 2001) found empirically that the latter example is significantly more difficult than the first. The most difficult problems, however, are equations of the type VI = VI + VI. This type of problem was consistently the most difficult problem in all our experiments, but why? The problem has two sources of problem difficulty. First, the constraint that operators are constants has to be relaxed and second, the more fundamental constraint that an equation per se consists of two different operators has to be overcome. After these insights the solution is trivial $(\mathrm{VI}=\mathrm{VI}=\mathrm{VI})$ - a tautological structure is necessary to solve the problem. Table 1 summarizes the theoretical assumptions and the empirical results of the Knoblich et al. $(1999,2001)$ studies. Note that solution rate is the inverse measure of problem difficulty. The more solutions to problems, the less difficult the problem is. 


\section{Table 1.}

Theoretical assumed solution rate of the different problem types. Italic font in the row Goal Representation indicates the locations in the equation that require changing. The gray shaded goal representation in the column Tautology indicates a deeper structural change of the equation. The number of ' + ' are a qualitative indicator of the expected solution rate. The empirical solution rates comprise the data found by Knoblich et al. (1999, 2001)

\begin{tabular}{|c|c|c|c|c|}
\hline Problem type & Value Type & Hybrid Type & Operator Type & Tautology \\
\hline Task & $\mathrm{IX}=\mathrm{VII}+\mathrm{VI}$ & $\mathrm{VI}=\mathrm{VI}+\mathrm{I}$ & $\mathrm{IV}=\mathrm{III}-\mathrm{I}$ & $\mathrm{IV}=\mathrm{IV}+\mathrm{IV}$ \\
\hline Solution & $\mathrm{XI}=\mathrm{VII}+\mathrm{IV}$ & $\mathrm{VI}=\mathrm{VII}-\mathrm{I}$ & $\mathrm{IV}-\mathrm{III}=\mathrm{I}$ & $\mathrm{IV}=\mathrm{IV}=\mathrm{IV}$ \\
\hline Goal Repres. & $\begin{array}{c}\operatorname{Var}_{1} \text { const }_{1} \operatorname{Var}_{2} \text { const }_{2} \\
\operatorname{Var}_{3}\end{array}$ & $\begin{array}{c}\operatorname{Var}_{1} \text { const }_{1} \operatorname{Var}_{2} O P \\
\operatorname{Var}_{3}\end{array}$ & $\begin{array}{c}\operatorname{Var}_{1} O P_{1} \operatorname{Var}_{2} O P_{2} \\
\operatorname{Var}_{3}\end{array}$ & $\begin{array}{c}\operatorname{Var}_{1}=\underset{\operatorname{Var}_{2}}{ } \mathrm{OP} \\
\operatorname{Var}_{3}\end{array}$ \\
\hline Solution rate & ++++ & +++ & ++ & + \\
\hline $\begin{array}{l}\text { Empirical } \\
\text { solution rates }\end{array}$ & $90 \%$ & $75 \%$ & $\sim 60 \%$ & $\sim 25 \%$ \\
\hline
\end{tabular}

\section{Test problems}

For the purpose of the present study, we constructed a new set of equations that required varying extents of constraint relaxation. Each problem was adapted so as to require the problem solver to move two matchsticks in order to transform an incorrect arithmetic statement into a correct one. We constructed two different types of test problems. The test problems always consisted of one value-move and one operator-move. For example, in the equation IV = III - III, the I of the IV is moved after the V to make VI = III - III, and then one matchstick from the $=$ is moved to make the - into an $=$, and so the solution VI - III = III is attained. Problems of this form are designated the Value-Operator type. The second type of test problems required the manipulation of a value and an operator to attain a tautological structure. For example, in the equation IV = IV + VI, the I from the value $V I$ is moved before the $V$, giving IV = IV + IV, and then the vertical matchstick from the + is moved to make the + into a second $=$, so the solution has the tautological structure IV = IV = IV - designated the Value-Tautology problem type (see Table 2).

The value-move is important - we already know from previous studies (e.g., Knoblich et al., 2001) that problem solvers will first of all look to change the values in matchstick arithmetic problems. We created two different sub-types of Value-Operator and ValueTautology problems, each consisting of a different kind of value-move. With this manipulation we attempted to test the assumptions of the Criterion for Satisfactory Progress Theory.

-Value (minus-value) problems consist of valuemoves that reduce the distance to the goal, supporting a pure hill-climbing heuristic. In contrast, +Value (plus-value) problems consist of value-moves that increase the distance to the goal, violating the hill- climbing heuristic of the Criterion for Satisfactory Progress Theory.

Let us exemplify this assumption: We assume that a perceived efficient heuristic to solve matchstick arithmetic tasks may be to compare both sides of the equation, and select moves that reduce this difference as much as possible. That is, confronting the problem solver with the equation IX = IV - III $(9=1)$ (a -Value-Operator problem) the difference between the both sides of the equation is 8 . The question is: How can the problem solver reduce this difference? She can do this by moving the stick behind the $V$ to be in front of it, IX = VI - III $(9=3)$. That is, this move reduces the distance between the left and the right side of the equation by 2 . This type of problem satisfies a hill-climbing strategy because the value-move reduces the distance to the goal.

In contrast, the equation IV = III - III $(4=0)$ (a +Value-Operator problem) has no available valuemove that reduces the distance to goal. The only available moves increase the distance to goal - such as VI $=$ III - III $(6=0)$. After this move, other value-moves are available (e.g., VII = III - II, $7=1$ ), but these also violate the hill-climbing heuristic. This type of problem does not satisfy a hill-climbing strategy because the value-move increases the distance to the goal.

We were also able to generate -Value-Tautology and +Value-Tautology problems, following the same construction rules. As stated previously, the problems chosen were all constraint relaxation problems, so one of the two solution-moves was a constraint relaxation move, which varied in difficulty (see Table 2 ).

It should be noted that it was not possible to control the amount of value moves that were possible for each equation. For example, the equation IX = VIII - III only has one value move available (the I before the X) whereas for the equation VI = IV + VI all of the ' $I$ ' matchsticks can be moved. This is a point that will 


\section{Table 2.}

Examples of the two-step matchstick arithmetic test problems, which entail a standard move (either -Value or +Value) and a constraint-relaxation (CR) move (Operator, or Tautology). -Value problems reduce the difference between the terms of the equation; +Value problems increase the difference (differences between the left and the right terms of the equation are shown in parentheses, with squared parentheses indicating the amount that a move increases or decreases the difference)

\begin{tabular}{|c|c|c|c|c|c|}
\hline Value type & CR type & Task & Value-move & CR-move & Solution \\
\hline -Value & Operator & $\begin{array}{c}\text { XIII }=\mathrm{IX}-\mathrm{VI} \\
13=3(10)\end{array}$ & $\begin{array}{c}\text { XIII }=\text { IX - IV } \\
13=5(8)[-2]\end{array}$ & $\begin{array}{l}\text { XIII }-I X=V I \\
4=6(2)[-8]\end{array}$ & $\begin{array}{c}\text { XIII - IX = IV } \\
4=4(0)\end{array}$ \\
\hline -Value & Tautology & $\begin{array}{l}\mathrm{IX}=\mathrm{XI}+\mathrm{IX} \\
9=20(11)\end{array}$ & $\begin{array}{c}\text { IX }=\text { IX + IX } \\
9=9+9(9)[-2]\end{array}$ & $\begin{array}{c}\mathrm{IX}=\mathrm{XI}=\mathrm{IX} \\
9=11=9(2)[-9]\end{array}$ & $\begin{array}{l}\text { IX }=\text { IX }=\text { IX } \\
9=9=9(0)\end{array}$ \\
\hline +Value & Operator & $\begin{array}{c}\text { IX }=\text { VIII - III } \\
9=5(4)\end{array}$ & $\begin{array}{c}X I=\text { VIII - III } \\
11=5(6)[+2]\end{array}$ & $\begin{array}{l}\text { IX - VIII = III } \\
1=3(2)[-2]\end{array}$ & $\begin{array}{c}X I-V I I I=I I I \\
3=3(0)\end{array}$ \\
\hline +Value & Tautology & $\begin{aligned} \mathrm{VI} & =\mathrm{IV}+\mathrm{VI} \\
6 & =10(4)\end{aligned}$ & $\begin{array}{c}\mathrm{VI}=\mathrm{VI}+\mathrm{VI} \\
6=12(6)[+2]\end{array}$ & $\begin{array}{c}\mathrm{VI}=\mathrm{IV}=\mathrm{VI} \\
6=4=6(2)[-2]\end{array}$ & $\begin{array}{l}V I=V I=V I \\
6=6=6(0)\end{array}$ \\
\hline
\end{tabular}

be returned to in the results section where a specific analysis that categorizes solution rates in relation to available value moves will show that the number of value moves available does not affect solution rates.

\section{Baseline problems}

We introduced two additional problem types as baseline conditions. These problem types serve as standard comparisons to the test problems, because both baseline problems require either no constraint relaxation or constraint relaxation to a lesser degree than the test problems.

Value-Value problems require no constraint relaxation, but can be solved by two subsequent valuemoves. For example, in order to solve III = XI - IV, there are two value-moves III $=$ IX - IV, and III $=$ XI - VI, so the result is III = IX - VI (see Table 3).

Value-Hybrid problems (see Tables 1 and 3) require both manipulating a value and moving a matchstick from an operator to a value. For example, in order to solve VI = IX + IV, the value-move changes IX into XI $(\mathrm{VI}=\mathrm{XI}+\mathrm{IV})$ and the operator/value move changes + into - and VI into VII (result: VII = XI - IV). This problem type can be characterized as a hybrid between the Value-Value and the Value-Operator types.

\section{Basic predictions from Criterion for Satisfactory Progress Theory}

According to the Criterion for Satisfactory Progress Theory, the problem solver should apply a maximising heuristic and therefore prefer moves that reduce the distance to goal (-Value problems in Table 2) to moves that increase the distance to goal (+Value problems in Table 2) (Chronicle et al., 2004). This prediction is also supported by studies that investigate the influence of hill-climbing on multi-step problems such as the Hobbit and Orcs problem (Greeno, 1974; Thomas, 1974), and the water jug problems (Atwood \& Polson, 1976). These studies demonstrated convincingly that when the problem solver was required to select moves that increased the distance to the goal, these moves were very time consuming and error-prone. The studies demonstrated that move selection is determined by the tendency to make the current state as similar to the desired goal state as possible - i.e., to reduce the distance (or difference) between the current state and the goal state as much as possible.

In terms of the Criterion for Satisfactory Progress Theory, problem solvers test against a maximization criterion, trying to reduce the difference between the left and the right term of the equation. Problems that

\section{Table 3.}

Examples of two-move matchstick arithmetic baseline problems (differences between the left and the right terms of the equation are shown in parentheses, with squared parentheses indicating the amount that a move increases or decreases the difference)

\begin{tabular}{lcccc}
\hline Problem Type & Task & Move 1 & Move 2 \\
\hline Value-Value & III $=$ XI - IV & III $=$ XI - VI & III = IX - IV & Solution \\
& $3=7(4)$ & $3=5(2)[-2]$ & $3=5(2)[-2]$ & $4=4(0)$ \\
Value-Hybrid & IX $=$ VIII - III & XI $=$ VIII - III & IX - VIII = III & XI - VIII = III \\
& $9=5(4)$ & $11=5(6)[+2]$ & $1=3(2)[-2]$ & $3=3(0)$ \\
\hline
\end{tabular}


require the selection of moves that initially increase the distance to the goal (i.e., +Value-Operator and +Value-Tautology problems) should be more difficult than problems where the selection of moves reduce the distance to the goal (i.e., -Value-Operator and -Value-Tautology problems). That is, the Criterion for Satisfactory Progress Theory expects a better performance for -Value problems than +Value problems.

It is important to note that the distance to goal manipulation as described above depends on the implicit assumption that the value-move is always selected before a constraint relaxation (CR) move (operator-, or tautology-move). We already know that problem solvers consider value moves first (e.g., Knoblich et al., 2001), but in addition, solution rates also support this assumption (see Table 1). Furthermore, as can be seen in Table 2, the CR moves in the -Value problems reduce the distance to goal substantially more than the CR moves in the +Value problems, so even if people made CR moves first, the Criterion for Satisfactory Progress Theory still predicts that -Value problems are easier. Nevertheless, an analysis of the type of first move that participants make will be made in the results in order to verify this assumption.

Note that all of the problems used in each subtype are of similar difficulty. In the -Value problems there is a similar distance to goal (-Value-Operator (10), -Value-Tautology (11)) and the same holds for the +Value condition (+Value-Operator (4), +ValueTautology (4)).

Taken together, the prediction of the Criterion for Satisfactory Progress Theory is that -Value problems are easier than +Value problems. The -Value problems not only reduce the distance to goal in their value moves (as opposed to increasing the distance for the +Value problems) but their CR moves also reduce the distance to the goal much further than the CR moves in the +Value problems. Therefore a major difference between the effects of the -Value and the +Value condition is predicted. Within each of the conditions, there should not be a difference across problem sub-types, because each of them reduces the distance to the goal by a similar amount.

\section{Basic predictions from the Representational Change Theory}

In contrast to the Criterion for Satisfactory Progress Theory, the Representational Change Theory suggests that the difference between the Value-Tautology and Value-Operator problems has nothing to do with the value-move (-Value, or +Value). That is, for the
Representational Change Theory, problem difficulty is governed by the amount of constraint relaxation the problem type requires, so there should be no difference in performance for -Value and +Value-moves.

According to the Representational Change Theory, problem difficulty of the matchstick problems outlined in Tables 1, 2 and 3 lies in the degree to which constraint relaxation is required. Four increasing levels of difficulty are given (Value-Value, Value-Hybrid, ValueOperator, Value-Tautology), with Value-Value being the easiest and Value-Tautology the hardest. Knoblich et al. (1999, 2001) have already found that value type problems are solved more often than operator type problems, which in turn are solved more often than the tautological problems (see Table 1). Subsequently, for the two-step matchstick arithmetic problems, because the same "amount of difficulty" is added to each problem type (Value, Hybrid, Operator, and Tautology), a similar pattern of solution rate is predicted: Value-Value > Value-Hybrid > Value-Operator $>$ Value-Tautology.

\section{Predictions relating to providing a partial-solution}

Participants find one-move matchstick problems difficult, and hence we assume that the solution of twomove matchstick arithmetic problems will also be difficult. A partial-solution will therefore be provided to the participants after a certain amount of time. Two different kinds of partial solution can be given: first, the value move can be shown, or second, the constraint relaxation move can be shown. Informing participants of one move reduces the problem to a standard one-move matchstick arithmetic task (see Table 1). This means that for the Representational Change Theory, providing a CR move should be more effective than providing a value move, because the $\mathrm{CR}$ move reduces the problem to a one-move value problem. Providing a value move reduces the problem to a constraint relaxation problem and hence the same pattern of solution rate should be found as for past research: Operator move > Tautology move (Knoblich et al., 1999; Knoblich et al., 2001; Öllinger \& Knoblich, 2003).

Predictions for the Criterion for Satisfactory Progress Theory are dependent upon the remaining move to be made after the partial solution move is given. If a value move is given as the partial solution move, then there is a CR move remaining. As can be seen in Table 2, the $C R$ moves in -Value problems reduce the distance to goal by a much greater amount than 
the CR moves in +Value problems. The Criterion for Satisfactory Progress Theory therefore predicts better performance for -Value problems than +Value problems when a value move is given as the partial solution move. If a $C R$ move is given as the partial solution move, the remaining value move always reduces the distance to goal by a factor of two, and so no difference across problems should be seen for CR moves as partial solution moves. In essence then, there should be a general performance benefit for-Value problems, but particularly for -Value problems when a value move is given as a partial solution.

\section{METHOD}

\section{Design}

A 2 (value move: -Value, +Value) x 2 (partial-solution type: value move, constraint relaxation move) between subjects design resulted in four experimental groups. All groups were shown the $(-/+$ Value $)-$ Tautology and (-/+Value)-Operator test problems and the two additional baseline problems (Value-Value and Value-Hybrid). The dependent variables were whether the problem was solved either before or after a partial solution, for each of the two blocks of problems.

\section{Move verification}

To verify the assumption that participants initially make value moves, the initial moves that participants made were recorded. Furthermore, we recorded how these moves altered the equation (i.e., whether they reduced the distance to the goal or increased it).

\section{Participants}

120 participants (37 male, 83 female; age range 17 36) were recruited by advertising at the University of Munich and in local newspapers and received $€ 12$ each. Participants were screened beforehand for familiarity with Roman numerals. Each participant was randomly assigned to one of the four experimental conditions and tested individually.

\section{Material and apparatus}

Twelve Roman numeral matchstick arithmetic problems were used, each of which could be solved by moving two matchsticks in order to transform a false arithmetic expression into a correct one. Each participant solved six problems, with only four problems pertaining to this study. The two remaining problems were so-called chunk decomposition problems that require a different representational change than constraint relaxation problems (see Knoblich et al., 1999, Ohlsson, 1992). To minimize the influence of particular problems, we used two sets of problems: Set $A$ and Set B. In table 2 the problems of Set $A$ are displayed. For Set $B$ we used similar problems that differ in their outline e.g. the Value-Tautology problems of Set B were IV = IV + VI and XI = IX + $X I$. For each participant a random order of problems was determined. Tables 2 and 3 give examples of the types of problem presented.

The study was fully computer-based using a program implemented in JAVA (SDK 1.3) and run on a PC using Windows 98 . The problems were displayed on a Belina 17" monitor.

\section{Procedure}

All participants were individually tested. Upon entering the lab, participants were seated in front of a computer screen and given instructions as to how the two-move matchstick arithmetic problems should be solved. The instructions noted: (1) that all problems could be solved by moving exactly two sticks; (2) that sticks could not be discarded; (3) that the only valid symbols were roman numerals and the arithmetic operators "+", "-", and "=". Furthermore, the participants were asked to record all their "ideas" and solution attempts in a text field for notes.

The program display consisted of four horizontal areas (see Figure 1). The topmost area presented the problem; the second area was provided for participants to make notes; the third area was used to present a partial solution move if the equation had not been solved after five minutes; and the lowermost area was used for participants to type in solutions to the problems. At the beginning of each trial the participants pressed an OK button at which point all text fields were erased and a new matchstick problem appeared. Six keys on the keyboard were labeled "I", "V", "X", "+", "-" and "=", and these were used for making notes and for entering proposed solutions. No other keys were functional during the experiment. Navigation across the areas was accomplished using a mouse.

Each participant worked on all six problems. The presentation of the problems was counterbalanced across participants. Participants were given ten minutes to solve each equation, unless they proposed a correct solution before this time limit. Immediate feedback was given regarding correct or incorrect solution attempts. If participants had not solved the 
equation after five minutes, the partial solution move gave either the value move or the constraint relaxation move (depending upon the experimental condition). If participants failed to solve an equation after ten minutes, they were shown the solution.

\section{RESULTS AND DISCUSSION}

We analyzed the results in terms of overall solution rates for each condition, solution rates before a partial solution move was given, and solution rates after a partial solution move. ANOVA was used for the majority of analyses. Although the data has binary characteristics, the use of ANOVA is warranted based on the theoretical assumptions by Greer and Dunlap (1997), and by its successful application by other authors (e.g., Knoblich et al., 1999; MacGregor et al., 2001). Finally, we conducted a move-analysis of the participants' move proposals and ideas recorded in the note field (see Figure 1 ). We classified the selected moves with respect to the applied moves of the equation (value move, hybrid move, or operator move), and calculated whether a selected move reduced or increased the distance to goal.

\section{Solution rates of the test problems before being given a partial solution move}

Figure 2 shows solution rates for the two test problems before a partial solution move was given for each of the -Value and +Value-conditions. A mixed ANOVA with the between-factor Value type (-Value, +Value) and the within-factor Problem type (Operator, Tautology) showed no main effect of Value type $[F(1,118)=1.54, p>.05]$. However, there was a highly significant main effect of Problem type $[F(1,118)=37.47, p<.001]$ with Operator problems being solved more often than Tautology problems. There was no reliable interaction between the two $[F(1,118)=.13, p>.05]$.

These findings suggest that the Value type manipulation did not influence problem difficulty, and that it was, in fact, the Problem type that drove problem difficulty. Thus, for matchstick arithmetic problems, people's performance does not seem to be influenced by the hill-climbing heuristic. This is further supported from the lack of an interaction between the Value type and Problem type variables. If both the Criterion for Satisfactory Progress Theory and Representational Change Theory had influenced problem solving, then one would expect problems in the -Value-Operator condition to be easiest, and problems in the +Value-

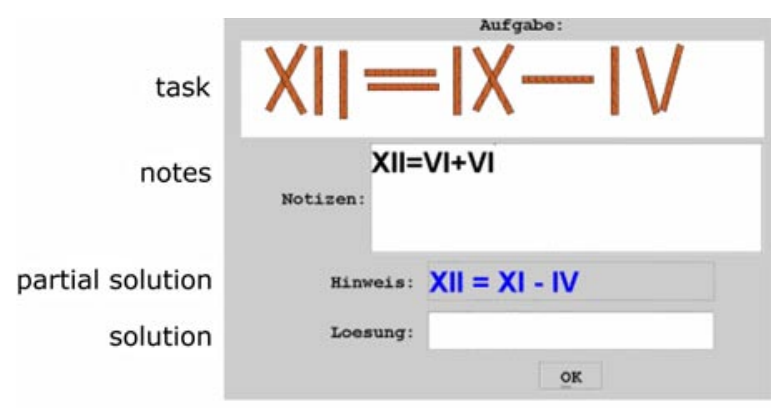

\section{Figure 1.}

Display of the user interface

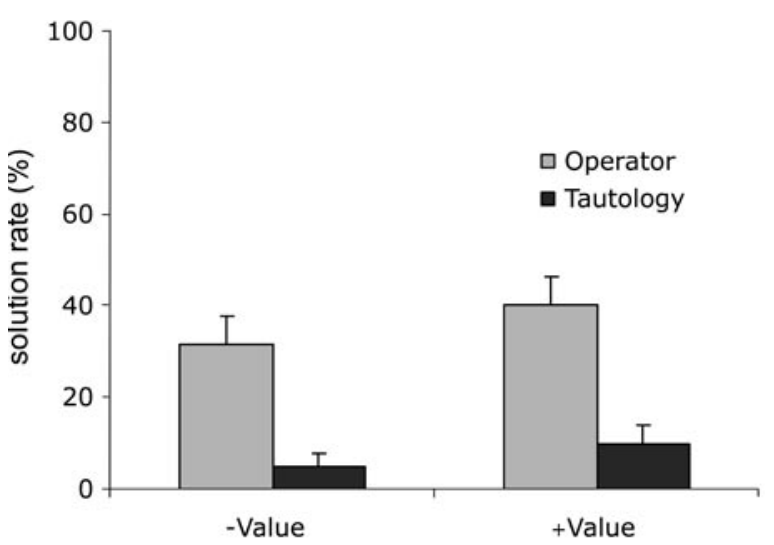

Figure 2.

Solution rates by condition before a partial-solution was provided

Tautology to be the most difficult (i.e., one would expect an interaction). The lack of a main effect of Value type, the lack of an interaction between Value type and Problem type, and the fact that there is a main effect of Problem type all lead to the conclusion that problem difficulty in these sets of problems depended on the type and level of constraint that needed to be relaxed.

\section{Solution rates of all problem types before being given a partial solution move}

Figure 3 illustrates the solution rates of the two baseline problems and the two test problems averaged across participants. A one-way ANOVA with the factor Problem type revealed a highly significant main effect $[F(1,357)=19.87, p<.001]$. Pairwise post-hoc comparisons revealed significant differences between all problem types ( $p<.05$ or better) except the ValueHybrid and Value-Operator type $(p>.05)$. This pattern of problem difficulty replicated, in principle, the findings of the one-move matchstick arithmetic domain (see Table 1). The Value-Value type was solved more often than any other problem type and the 


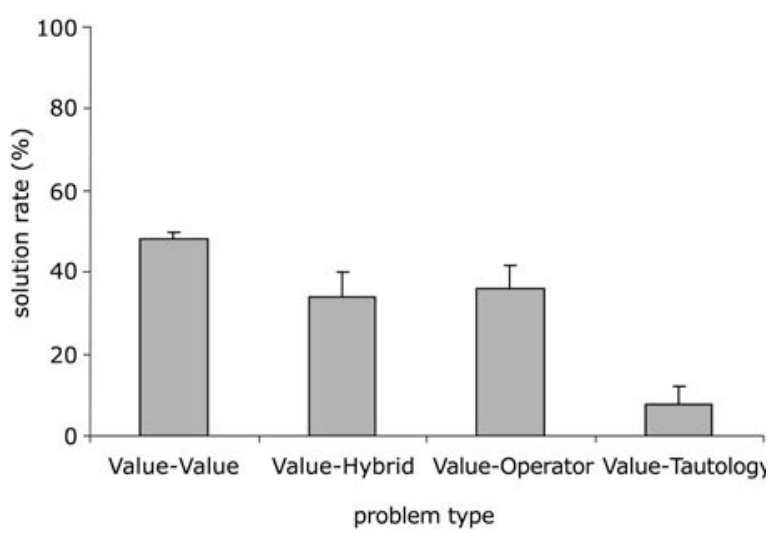

Figure 3.

Problem difficulty of test and baseline problems before a partial solution was given

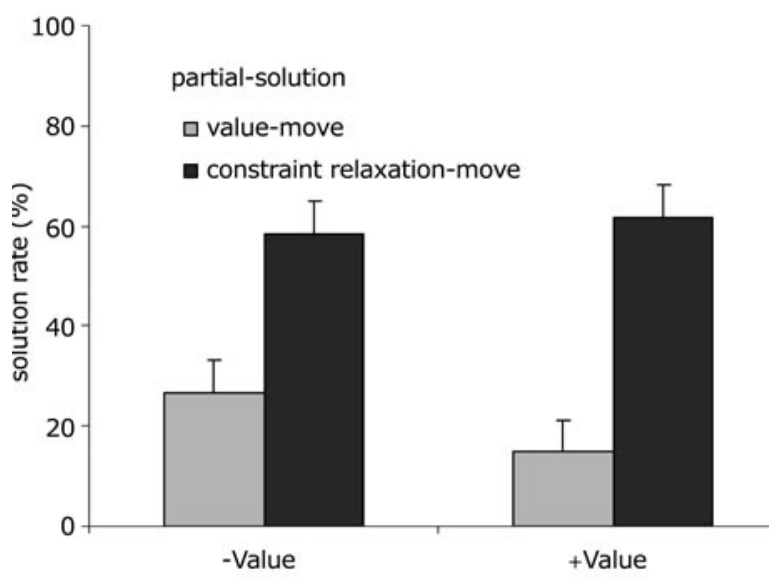

Figure 4.

Solution rates of the test problems after providing a partialsolution. Solution rates are broken down by Value-type

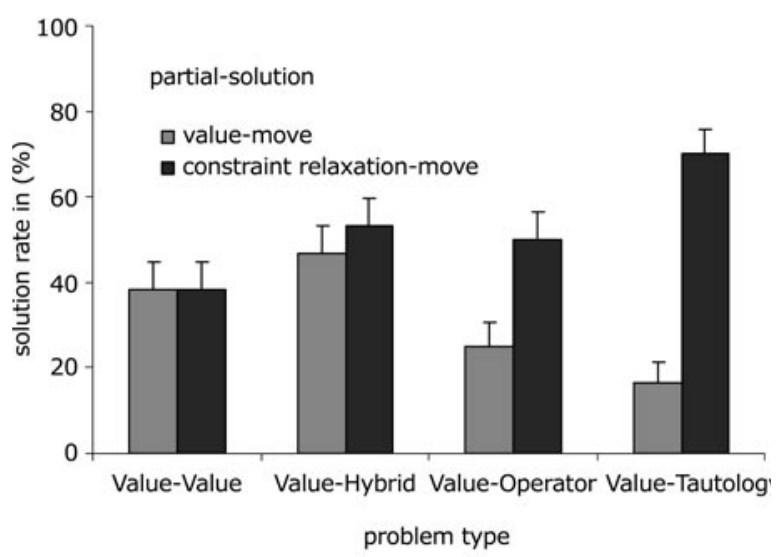

Figure 5.

Solution rates of the test and baseline problems after providing a partial-solution. Solution rates are broken down by Problem-type

Value-Tautology was by far the most difficult problem type. Value-Hybrid and Value-Operator problems lay in between. The solution rate of two-move matchstick arithmetic problems depended on the constraints that needed to be relaxed.

\section{Solution rates of the test problems after being given a partial solution move}

Figure 4 shows solution rates for the test problems after a partial solution was given, broken down by Value type. The figure clearly demonstrates the benefit of giving a CR move over a value move as a partial solution move. A mixed ANOVA with the two between-factors Value type and Partial solution type and the within-factor Problem type showed a highly significant main effect of Partial solution type $[F(1,116)=45.15, p<.001]$ with the $\mathrm{CR}$ moves proving more beneficial to solution rates than the value moves (see Figure 4). There were no main effects of Value type $[F(1,116)=0.51, p>.05]$ or Problem type $[F(1,116)=1.06 ; p>.05]$. However, there was a significant interaction between Partial solution type and Problem type $[F(1,116)=6.24$, $p<.05]$. That is, the deeper the level of constraint that was relaxed by the partial solution, the more effective the partial solution was. There were no further significant interactions between any combinations of the factors $(p>.05)$.

The results support the partial solution move predictions of the Representational Change Theory, which suggested that giving people the CR move would be more beneficial than the value move. The results fail to support the predictions of the Criterion for Satisfactory Progress Theory. There was no difference between Value and +Value groups, and there was no significant interaction between value type and partial solution type $[F(1,116)=1.66, p>.05]$. Solution rates were not higher in the -Value condition when people were given the value move as the partial solution move.

Figure 5 shows all problem types (baseline and test problems; note that for the Value-Value problem, of course, no CR move exists, therefore this problem type is not included in the statistical analysis). A mixed ANOVA with the between-factor Partial solution move (Value move, CR move) and the within-factor Problem type (Value-Hybrid, Value-Operator, Value-Tautology) revealed a highly significant main effect for the factor Partial solution move $[F(1,118)=31.20, p<.001]$ with the $C R$ move providing greater benefit to solution rates than the value move. Furthermore, there was a highly significant interaction between the factors $[F(2,236)=7.87, p<.001]$. There was no main effect of Problem type $[F(2,236)=2.23, p>.05]$. These results show (as do the black colored columns 
Table 4.

Frequency of different moves that either did not change, increased, or reduced the amount between the left and the right side of the equation

\begin{tabular}{lccc}
\hline & neutral-moves & increase-moves & reduce-moves \\
\hline -Value & $21(4.13 \%)$ & $13(2.55 \%)$ & $248(48.72 \%)$ \\
+Value & $24(4.72 \%)$ & $16(3.14 \%)$ & $187(36.74 \%)$ \\
total & $45(8.85 \%)$ & $29(5.69 \%)$ & $435(85.46 \%)$ \\
\hline
\end{tabular}

in Figure 5) that the greater the degree of constraint relaxation a problem type requires, the greater the benefit of giving that move as a partial solution move. Giving a value move as partial solution move results in a pattern that is similar to the one-move results (as illustrated by the gray colored columns in Figure 5; see also Table 1 as a reference). For the Value-Value type the participants benefit equally, as would be expected. Pairwise post-hoc comparisons for the value move as partial solution condition revealed highly significant differences between the Value-Hybrid and the ValueOperator problem types $(p<.001)$. There was no significant difference between the Value-Operator and the Value-Tautology problems $(p>.05)$.

In general the results show that, not surprisingly, providing a partial solution move facilitates the solution of the problems. However, solution rates clearly depend on the move type - CR moves are much more effective than value moves, at least for the test problems in this study. This finding can be summarized: the more "difficult" the constraint that a partial solution relaxes, the more a problem solver benefits from the partial solution.

\section{Move verification}

At the outset of this investigation, it was assumed that people have an initial preference for value moves. First, this was supported from previous studies (e.g., Knoblich et al., 1999, 2001) and second, this was assumed for the predictions of the Criterion for Satisfactory Progress Theory, although the predictions were not actually dependent on it. In order to check the preference for value moves, we analyzed the data recorded in the note field (see Figure 1), where the participants were asked to key in all their ideas, proposals, and solution attempts. We analyzed the given proposals by counting up the type of manipulations that participants applied. In total, there were 690 inputs in the note field. 181 inputs were invalid, that is, the inputs were not proper equations (either having a wrong number of values and/or operators). Of the remaining 509 equations, $60.89 \%$ were value moves, $22.47 \%$ moves were hybrid moves (moves between an operator and a value), and $16.64 \%$ were operator moves. Even when combining the hybrid moves and operator moves, a $x^{2}$-test showed that significantly more value moves were made than other types of moves $\left[\chi^{2}(1)=24.21, p<.001\right]$.

Another area of interest is the direction of the move (whether it reduces or increases the distance to the goal). The Criterion for Satisfactory Progress Theory predicts that people make moves that reduce the distance to the goal because they apply a hill-climbing heuristic. Table 4 illustrates the distribution of the move types participants used, broken down by the experimental conditions (-Value condition and +Value condition). The analysis revealed an additional move type, labeled neutral moves that do not change the difference between the two sides of the equation. As Table 4 shows, in the vast majority of cases, participants selected moves that reduced the distance to the goal - supporting the prediction from the Criterion for Satisfactory Progress Theory.

We conducted $\chi^{2}$-tests for both - Value and +Value conditions. Even when combining neutral moves and increase moves, the analysis showed that people made significantly more reduce moves than other types of move, for both the -Value condition $\left(\chi^{2}(2)=162.40\right.$, $p<.001)$ and the +Value condition $\left(\chi^{2}(1)=95.19\right.$, $p<.001)$. A further analysis compared the participants' reduce move preference between the two conditions (-Value and +Value). A $\chi^{2}$-analysis revealed a significant effect, $\chi^{2}(1)=8.55, p=.01$, that is, people in the -Value condition had a higher frequency of selecting moves that reduce the distance to the goal than people in the +Value condition.

\section{Number of value moves available}

As stated earlier, we were not able to control the number of available value moves that participants could make in the problems given, and it is conceivable that performance systematically varied with the size of the given problem space. Therefore, we categorized all problems with respect to their number of available value moves for the first move and determined the average performance of each category. Figure 6 plots the categories and the assigned performance. As can 


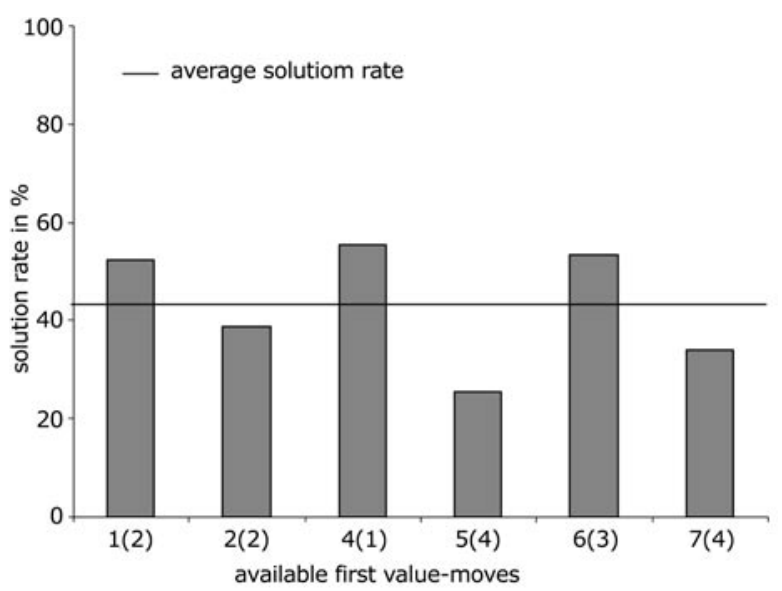

Figure 6.

Solution rates of the test and baseline problems after providing a partial-solution. Solution rates are broken down by Problem-type

be seen, there was no systematic increase or decrease in performance based on this factor. A linear regression with the independent variable number of available first value moves and the dependent variable solution rate revealed a non-significant regression coefficient $R=.30,(p=.56)$, illustrating that the number of available value moves as a first move did not affect solution rates.

\section{The influence of problem sets $A$ and $B$ on the solution rate}

As mentioned above, we used two different sets of problems, A and B. Table 5 illustrates the solution percentages for the problems in sets $A$ and $B$. The two sets revealed quite similar results. Again the solution rate varied by and large with the extent of the required constraint relaxation. A $\chi^{2}$-analysis between the two sets revealed no significant differences $(p>.10)$.

\section{GENERAL DISCUSSION}

In the present study we were interested in the possible interplay of heuristics and representational change when solving insight problems. We aimed to contribute to the question whether the main source of problem difficulty of insight problems results from the use of inappropriate heuristics (MacGregor et al., 2001), or from the constraints that problem solvers impose on a given problem (Ohlsson, 1992; Knoblich et al., 1999), or as a third possibility, whether both sources have an influence (Jones, 2003). In four groups we varied systematically the efficiency of heuristics and the degree of necessary constraint relaxation. Our experimental manipulation did not reveal any significant influence of heuristics, going against the predictions of the Criterion for Satisfactory Progress Theory. In contrast the solution rate was clearly driven by the degree of representational change, as predicted by the Representational Change Theory.

Furthermore, there was, in general, a clear pattern of problem difficulty (see Figure 2 and Figure 3) that was rather similar to the findings of Knoblich et al. (1999) in the single matchstick arithmetic domain (see Table 1). The more flexible the goal representation has to be, the more difficult the problem becomes (Value-Tautology $>$ Value-Operator > Value-Hybrid > Value-Value; read ">" as "more difficult than"). Importantly, solution rates for the Value-Operator problems were essentially higher than for the Value-Tautology problems. These findings can only be explained in terms of the necessity of a representational change being crucial for the solution of insight problems, and argues against the assumption that problem solvers prefer a hill-climbing approach that reduces the distance to goal as much as possible (Chronicle et al., 2004).

The results also showed that there was no difference across the -Value and +Value groups in terms of solution rates. Performance was not affected differentially by value moves that fulfill a maximization criterion that reduces the distance to goal, in comparison to value moves that increase the distance to goal. Under the internal criterion of selecting moves that reduce the distance to goal, the Criterion for Satisfactory Progress Theory would predict that when solution moves fulfill this criterion, performance should be improved over when solution moves do not fulfill this criterion. Such predictions have not been borne out by the data presented here.

Examining the baseline problems and the test problems, the Representational Change Theory claims that a more flexible goal representation makes operator

Table 3.

Solution rates of the different problem types broken down by problem set

\begin{tabular}{lcccc}
\hline & Value-Value & Value-Hybrid & Value-Operator & Value-Tautology \\
\hline Set A & 48.3 & 36.67 & 31.67 & 3.34 \\
Set B & 48.3 & 31.67 & 40.0 & 11.67 \\
\hline
\end{tabular}


problems significantly more difficult than hybrid problems. Interestingly, there was no difference between Value-Operator and Value-Hybrid problems. However, after a value move was given as a partial solution, operator problems were significantly more difficult than hybrid problems, fitting the predictions from the Representational Change Theory. Clearly, making the matchstick problem a two-move problem increases the size of the problem space, and this has affected the influence of a more flexible goal representation on the solution rate. A larger problem space and search space had more impact on the problem difficulty than the differences in the necessary goal representation. This is a potential moment where heuristics could become important, because as the problem space becomes larger, people need effective heuristics that help to constrain the given problem space (Kaplan \& Simon, 1990).

As expected from both theories, providing a partial solution move reduced the problem difficulty. Providing either a value move or a CR move increased the solution rates, because providing part of the solution reduces the problem space. The asymmetry in the performance after providing a partial solution move supported the Representational Change Theory. A CR move was much more effective than a value move. CR moves reduced, according to the assumptions of the Representational Change Theory, the main source of problem difficulty. CR moves provided the problem solvers with a more flexible goal representation than value moves did. In principle, we also found the predicted pattern of problem difficulty after giving a value move as the partial solution move - this reduces the problem to a onemove problem whereby the pattern of problem difficulty should be the same as that of previous literature (i.e., the more difficult the constraint to relax, the more difficult the problem is to solve). That is, the necessity of a more or less flexible goal representation drives problem difficulty. After a value-move being given, therefore, the solution rate of Value-Hybrid problems was, as expected, higher than the solution rate of Value-Operator problems. Value-Hybrid problems were also easier than the Value-Tautology problems.

The predictions of the Criterion for Satisfactory Progress Theory with regard to providing partial solution moves were not supported. The Criterion for Satisfactory Progress Theory argues that when a partial solution move is provided, the -Value condition should benefit more than the +Value condition. Specifically, if a value move is given as the partial solution move, then the -Value condition should show an increase in solution rates over the +Value condition, because the remaining $C R$ move reduces the distance to the goal more for the -Value problems. None of the results supported these predictions.

A point worth mentioning is that the experimental design actually works somewhat against the hypotheses of the Representational Change Theory, because there could have been a positive transfer due to the partial solutions (see Knoblich et al., 1999). Imagine the hypothetical case that a participant in the value move partial solution condition was confronted with the Value-Tautology problem type as her first problem and she could not solve it. After the upper time limit the solution was provided to her, and after obtaining this information she knew that the manipulation of operators and the changing of the common structure of an equation were appropriate means to solve a matchstick arithmetic problem. Such knowledge should provide her with an advantage when solving the remainder of the matchstick problems. Taking this into account, the findings presented here actually exceed our expectations.

The analyses of the solution attempts and proposals keyed in the note field showed clearly that people preferred moves that manipulate values, as expected by the prior knowledge hypothesis of the Representational Change Theory. Furthermore, people preferred the selection of moves that actually reduce the distance to goal, as proposed by the Criterion for Satisfactory Progress Theory. Both findings support our experimental logic and gave us further insight into the processes that are involved in insight problem solving.

As often claimed by Knoblich and colleagues (1999, 2001) some matchstick arithmetic problems are insight problems because they require the problem solver to overcome prior knowledge. This follows the tradition of the Gestaltists who were the first to see the relationship between the hampering influence of prior knowledge and the difficulty of solving problems that actually require overcoming such knowledge (Duncker, 1945; Maier, 1930, 1931; Luchins, 1942; Wertheimer, 1959; see also Ohlsson, 1984a, 1984b). In the matchstick arithmetic task, prior knowledge means that people have learned to solve arithmetic equations by changing the values of the equation. Now we can confirm this assumption, because more than $60 \%$ of the moves made were value-moves, although people learned more and more operator moves during the experiment (see above). Moreover, we can also confirm the Criterion for Satisfactory Progress Theory assumption that people prefer moves that reduce the distance to goal. Almost all selected moves reduced the distance to goal. This preference was in principle independent from the experimental conditions, that is, whether problem solvers belonged to the -Value 
or +Value conditions. However, as mentioned before, there is no indication that this preference influenced the probability of solving an insight problem. In general, the findings presented here suggest that the problem difficulty of two-move matchstick arithmetic tasks is not a result of the application of inappropriate heuristics, but the result of an inappropriate problem representation.

The Representational Change Theory provides an explanation of the insight process that fits in with the findings presented here much better than the Criterion for Satisfactory Progress Theory. The remaining question is whether the Criterion for Satisfactory Progress Theory and Representational Change Theory can both contribute to the comprehension and explanation of insight problems. The link between the two theories is the fact that a heuristic is likely to be worthless unless it is applied to an appropriate problem representation (Kaplan \& Simon, 1990). While the current paper has attempted to demarcate the two theories, a more suitable approach may be to suggest that the Criterion for Satisfactory Progress Theory explains the phase before an impasse, where inappropriate heuristics are performed on an inappropriate representation, and the Representational Change Theory explains the phase within an impasse where the representation is changed such that more appropriate heuristics can be applied. Jones (2003) came to a similar conclusion - that is, insight (the representational change) opens the door for the appropriate application of well known strategies (heuristics).

This way of thinking also accounts for the findings presented here. In our view insight depends on an underlying representational change, whereby the process of a representational change is by and large an unconscious process that seems obviously non-stepwise, sudden, and discontinuous (Bowden, 1997; Bowden et al. 2005, Bowden \& Beeman, 1998; Bowden \& JungBeeman, 2003; Grant \& Spivey, 2003; Jung-Beeman et al., 2004; Metcalfe, 1986a, 1986b; Metcalfe \& Wiebe, 1987; Wegner, 2002). The representational change is the door opener that ensures that the appropriate heuristics can be applied to the proper problem representation. The influence of heuristics on insight can sometimes be more important (MacGregor et al., 2001; Ormerod et al., 2002; Chronicle et al., 2004) yet at the same time, it can occasionally play no major role, as in the study presented.

\section{References}

Anderson, J. R. \& Lebiere, C. (1998). The atomic components of thought. Mahwah, NJ: Erlbaum.
Atwood, M. E. \& Polson, P. G. (1976). A process model for water jug problems. Cognitive Psychology, 8, 191-216. WWw

Bowden, E. M. (1997). The effect of reportable and unreportable hints on anagram solution and the aha! experience. Consciousness \& Cognition, 6, 545-573. [WWW

Bowden, E. M. \& Beeman, M. J. (1998). Getting the right idea: Semantic activation in the right hemisphere may help solve insight problems. Psychological Science, 9, 435-440.

Bowden, E. M. \& Jung-Beeman, M. (2003). Aha! Insight experience correlates with solution activation in the right hemisphere. Psychonomic Bulletin \& Review, 10, 730-737. www

Bowden, E. M., Jung-Beeman, M., Fleck, J. \& Kounios, J. (2005). New approaches to demystifying insight. Trends in Cognitive Sciences, 9, 322-328. |Ww|

Bühler, K. (1907). Tatsachen und Probleme zu einer Psychologie der Denkvorgänge. Archiv für Psychologie, 9, 297-365.

Chronicle, E. P., MacGregor, J. N. \& Ormerod, T. C. (2004). What Makes an Insight Problem? The Roles of Heuristics, Goal Conception, and Solution Recoding in Knowledge-Lean Problems. Journal of Experimental Psychology: Learning, Memory, \& Cognition January, 30, 14-27.

Duncker, K. (1945). On problem-solving. Psychological Monographs, 58, ix, 113.

Gigerenzer, G. \& Todd, P (1999). Simple heuristics that make us smart. Oxford: Oxford Univ. Press.

Grant, E. R. \& Spivey, M. J. (2003). Eye movements and problem solving: Guiding attention guides thought. Psychological Science, 14, 462-466.

Greeno, J. (1974). Hobbits and Orcs - acquisition of a sequential concept. Cognitive Psychology, 6, 270-292.

Greer, T. \& Dunlap, W. P. (1997). Analysis of variance with ipsative measures. Psychological Methods, 2, 200-207.

Gruber, H. E. (1995). Insight and affect in the history of science. In: Sternberg, R. J. \& Davidson, J. E. (Eds.), The nature of insight (p. 397-431). Cambridge, MA: MIT Press.

Isaak, M. I. \& Just, M. A. (1995). Constraints on thinking in insight and invention. In: Sternberg, R. J. \& Davidson, J. E. (Eds.), The nature of insight (p. 281325). Cambridge, MA: MIT Press.

Jones, G. (2003). Testing two cognitive theories of insight. Journal of Experimental Psychology: Learning, Memory, \& Cognition, 29, 1017-1027. |WWW|

Jung-Beeman, M., Bowden, E., Haberman, J., Frymiare, J., Arambel-Liu, S., Greenblatt, R., et al. (2004). 
Neural activity when people solve verbal problems with insight. Plos Biology, 2, 500-510. |WWw

Kaplan, C. A. \& Simon, H. A. (1990). In search of insight. Cognitive Psychology, 22, 374-419.

Katona, G. (1940). Organizing and memorizing: studies in the psychology of learning and teaching. New York: Columbia University.

Kershaw, T. C. \& Ohlsson, S. (2004). Multiple Causes of Difficulty in Insight: The Case of the Nine-Dot Problem. Journal of Experimental Psychology: Learning, Memory, \& Cognition January, 30, 3-13. |Www

Knoblich, G., Ohlsson, S., Haider, H. \& Rhenius, D. (1999). Constraint relaxation and chunk decomposition in insight problem solving. Journal of Experimental Psychology: Learning, Memory \& Cognition, 25, 1534-1555. www

Knoblich, G., Ohlsson, S. \& Raney, G. E. (2001). An eye movement study of insight problem solving. Memory \& Cognition, 29, 1000-1009. WwW

Lovett, M. C. \& Anderson, J. R. (1996). History of success and current context in problem solving: Combined influences on operator selection. Cognitive Psychology, 31, 168-217.

Luchins, A. S. (1942). Mechanization in problem solving-the effect of Einstellung. Psychological Monographs, 54, 1-95.

MacGregor, J. N., Ormerod, T. C. \& Chronicle, E. P. (2001). Information processing and insight: A process model of performance on the nine-dot and related problems. Journal of Experimental Psychology: Learning, Memory, \& Cognition, 27, 176-201. |WwW

Maier, N. R. F. (1930). Reasoning in humans. I. On direction. Journal of Comparative Psychology, 10, 115-143.

Maier, N. R. F. (1931). Reasoning in humans. II. The solution of a problem and its appearance in consciousness. Journal of Comparative Psychology, 12, 181-194.

Mayer, R. E. (1992). Thinking, problem solving, cognition (2nd ed.). New York: W. H. Freeman and Company.

Metcalfe, J. (1986a). Feeling of knowing in memory and problem solving. Journal of Experimental Psychology: Learning, Memory, \& Cognition, 12, 288-294. |www
Metcalfe, J. (1986b). Premonitions of insight predict impending error. Journal of Experimental Psychology: Learning, Memory, \& Cognition, 12, 623-634.

Metcalfe, J. \& Wiebe, D. (1987). Intuition in insight and noninsight problem solving. Memory \& Cognition, 15, 238-246.

Newell, A. \& Simon, H. A. (1972). Human problem solving. Englewood Cliffs, NJ: Prentice Hall.

Novick, L. R., and Sherman, S.J. (2003). On the nature of insight solutions: Evidence from skill differences in anagram solution. Quarterly Journal of Experimental Psychology Section A-Human Experimental Psychology, 56, 351-382.

Ohlsson, S. (1984a). Restructuring revisited: I. Summary and critique of the Gestalt theory of problem solving. Scandinavian Journal of Psychology, 25, 65-78.

Ohlsson, S. (1984b). Restructuring revisited: II. An information processing theory of restructuring and insight. Scandinavian Journal of Psychology, 25, 117-129.

Ohlsson, S. (1992). Information-processing explanations of insight and related phenomena. In: Keane, M. \& Gilhooly, K. (Eds.), Advances in the psychology of thinking (p. 1-44). London: HarvesterWheatsheaf.

Öllinger, M. \& Knoblich, G. (2003). Prioritizing the new: Insight creates set for familiar problems. In: Schmalhofer, F. \& Young, R. (Eds.), Proceedings of the First European Cognitive Science Conference 2003 (p. 253-258). Mahwah: NJ: Erlbaum.

Ormerod, T. C., MacGregor, J. N. \& Chronicle, E. P. (2002). Dynamics and constraints in insight problem solving. Journal of Experimental Psychology: Learning, Memory, \& Cognition, 28, 791-799. Www

Sternberg, R. J. \& Davidson, J. E., Eds. (1995). The nature of insight. Cambridge, MA: MIT Press.

Thomas, J. C. (1974). An analysis of behavior in the hobbits-orcs problem. Cognitive Psychology, 6, 257 269.

Wegner, D. M. (2002). The illusion of conscious will. Cambridge, MA: MIT Press.

Wertheimer, M. (1959). Productive thinking. New York: Harper. 